\title{
Identification and characterization of circadian clock genes in a native tobacco, Nicotiana attenuata
}

Felipe Yon ${ }^{1 \dagger}$, Pil-Joon Seo ${ }^{2,3 \dagger}$, Jae Yong Ryu ${ }^{2}$, Chung-Mo Park ${ }^{2}$, lan T Baldwin ${ }^{1}$ and Sang-Gyu Kim ${ }^{1 *}$

\begin{abstract}
Background: A plant's endogenous clock (circadian clock) entrains physiological processes to light/dark and temperature cycles. Forward and reverse genetic approaches in Arabidopsis have revealed the mechanisms of the circadian clock and its components in the genome. Similar approaches have been used to characterize conserved clock elements in several plant species. A wild tobacco, Nicotiana attenuata has been studied extensively to understand responses to biotic or abiotic stress in the glasshouse and also in their native habitat. During two decades of field experiment, we observed several diurnal rhythmic traits of $N$. attenuata in nature. To expand our knowledge of circadian clock function into the entrainment of traits important for ecological processes, we here report three core clock components in N. attenuata.
\end{abstract}

Results: Protein similarity and transcript accumulation allowed us to isolate orthologous genes of the core circadian clock components, LATE ELONGATED HYPOCOTYL (LHY), TIMING OF CAB EXPRESSION 1/PSEUDO-RESPONSE REGULATOR 1 (TOC1/PRR1), and ZEITLUPE (ZTL). Transcript accumulation of NaLHY peaked at dawn and NaTOC1 peaked at dusk in plants grown under long day conditions. Ectopic expression of NaLHY and NaZTL in Arabidopsis resulted in elongated hypocotyl and late-flowering phenotypes. Protein interactions between NaTOC1 and NaZTL were confirmed by yeast two-hybrid assays. Finally, when NaTOC1 was silenced in N. attenuata, late-flowering phenotypes under long day conditions were clearly observed.

Conclusions: We identified three core circadian clock genes in $\mathrm{N}$. attenuata and demonstrated the functional and biochemical conservation of NaLHY, NaTOC1, and NaZTL.

Keywords: Circadian clock, Flowering time, NaLHY, NaTOC1, NaZTL, Nicotiana attenuata, Protein interaction

\section{Background}

The circadian clock, entrained by our planet's $24 \mathrm{~h}$ rotation on its tilted axis, plays crucial roles in the synchronization of the performance of organisms with daily cycles of light and temperature, enabling organisms to regulate activities at the correct time of a day [1]. For instance, the endogenous clock in plants influences various biological processes including leaf movements, hypocotyl growth, floral transition, and abiotic and biotic stress resistance [2-4].

\footnotetext{
* Correspondence: skim@ice.mpg.de

${ }^{\dagger}$ Equal contributors

'Department of Molecular Ecology, Max Planck Institute for Chemical

Ecology, Hans-Knöll-Straße 8, Jena D-07745, Germany

Full list of author information is available at the end of the article
}

The circadian rhythmicity and molecular mechanisms underlying the circadian clock have been investigated in many organisms including Drosophila melanogaster, Neurospora crassa, Synechoccocus elongatus, and mice [5-7]. In general, several interconnected transcription/ translation feedback loops participate to establish central clock oscillations [8-10]. In plants, circadian rhythmicity is extensively investigated in a dicotyledonous model plant, Arabidopsis thaliana, and a 'three-loop model' has been proposed [11].

TIMING OF CAB EXPRESSION 1/PSEUDO-RESPONSE REGULATOR 1 (TOC1/PRR1) and two partially redundant MYB transcription factors, CIRCADIAN CLOCKASSOCIATED 1 (CCA1) and LATE ELONGATED HYPOCOTYL (LHY) comprise a central oscillation loop [12]. CCA1 and LHY repress transcript expression of

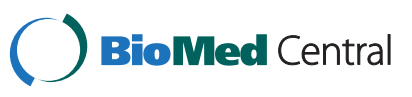


TOC1 by binding directly to its promoter, and as shown recently TOC1 negatively regulates the transcription of $C C A 1$ and $L H Y[13,14]$, establishing negative feedback loop $[11,15]$. The second loop is formed by TOC1 and the evening complex (EC) consisting of EARLY FLOWERING 3 (ELF3), ELF4, and LUX ARRHYTHMO (LUX) [14]. In addition, the third loop is established by negative feedback between PRR7/PRR9 and LHY/CCA1 [16,17].

Protein turnover regulation provides another layer of sophistication in the regulation of the circadian clock. Notably, the TOC1 protein is regulated by proteasomemediated protein degradation. The F-box protein ZEITLUPE (ZTL) in the E3 ubiquitin ligase SCF (SKP1CUL1-F-box protein) complex ubiquitinates the TOC1 protein through direct physical interaction in a darkdependent manner [18]. Homologs of ZTL are also involved in circadian regulation of photoperiodic flowering $[19,20]$. The FLAVIN-BINDING, KELCH REPEAT, F-BOX 1/ADAGIO3 (FKF1/ADO3) interacts with GI in a blue light-dependent manner and regulates $C O N$ STANS expression by degrading a Dof transcription factor, CYCLING DOF FACTOR 1 [19]. In addition, it has been reported that ZTL, FKF1, and LOV KELCH PROTEIN 2 together regulate TOC1 and PRR5 degradation, contributing to the circadian oscillation [21].

Recent works has shown that many of these circadian clock components can also be found in diverse plant species including rice, soybean, maize, and poplar [2225]. CCA1/LHY genes are widely conserved in eudicotyledonous (eudicots) and monocotyledonous (monocots) plants [26-29], and CCA1/LHY and TOC1 feedback loops are thought to play a central role in the clock's function in these plant species. Functional homologs of ZTL have also been found in several plant species $[30,31]$, further supporting that circadian clock components are fairly well-conserved in plants.

Despite the important role of the endogenous clock in entraining physiological processes to environmental signals, how the circadian clock regulates ecological performance of a plant in its natural habitat is largely unknown. Only a few studies have shown that the endogenous clock allows plants to maximize photosynthetic capacity and reproductive success [32-34]. In order to expand our understanding of the clock function in biotic and abiotic interactions, we identified three core clock components (LHY, TOC1, and ZTL) in a wild tobacco, Nicotiana attenuata, which has been developed as a model system for understanding ecological performance in native habitats, in particular the Great Basin desert in Utah. N. attenuata is a seasonal solanaceous plant, completing its life cycle during spring and summer to lie dormant in the seed bank for the many years between fires in its native habitat. These results provide additional evidence of the conservation of the circadian clock genes and set the stage for future studies to unravel the ecological relevance of the clock.

\section{Results}

\section{Isolation of putative core circadian clock genes in} N. attenuata

The full-length or partial sequences of three putative core circadian clock genes (LHY, TOC1, and ZTL) in N. attenuata were isolated by BLAST search against inhouse cDNA library using the sequences of Arabidopsis clock genes. We checked the diurnal expression of these transcripts in our time series microarray database [35], which examined patterns of transcript accumulation in $N$. attenuata leaf and root tissues every $4 \mathrm{~h}$ for one day (Figure 1). To confirm the microarray data and examine circadian rhythms of the selected clock genes in $N$. attenuata, we analyzed the transcript accumulation of the candidate genes in seedlings grown under different light conditions. $N$. attenuata plants were grown under $16 \mathrm{~h}$ light/ $8 \mathrm{~h}$ dark cycle (LD) for two weeks and subsequently transferred into continuous light condition (LL). Twenty seedlings in LD and LL were harvested every $2 \mathrm{~h}$ for three days. Quantification of mRNA expression

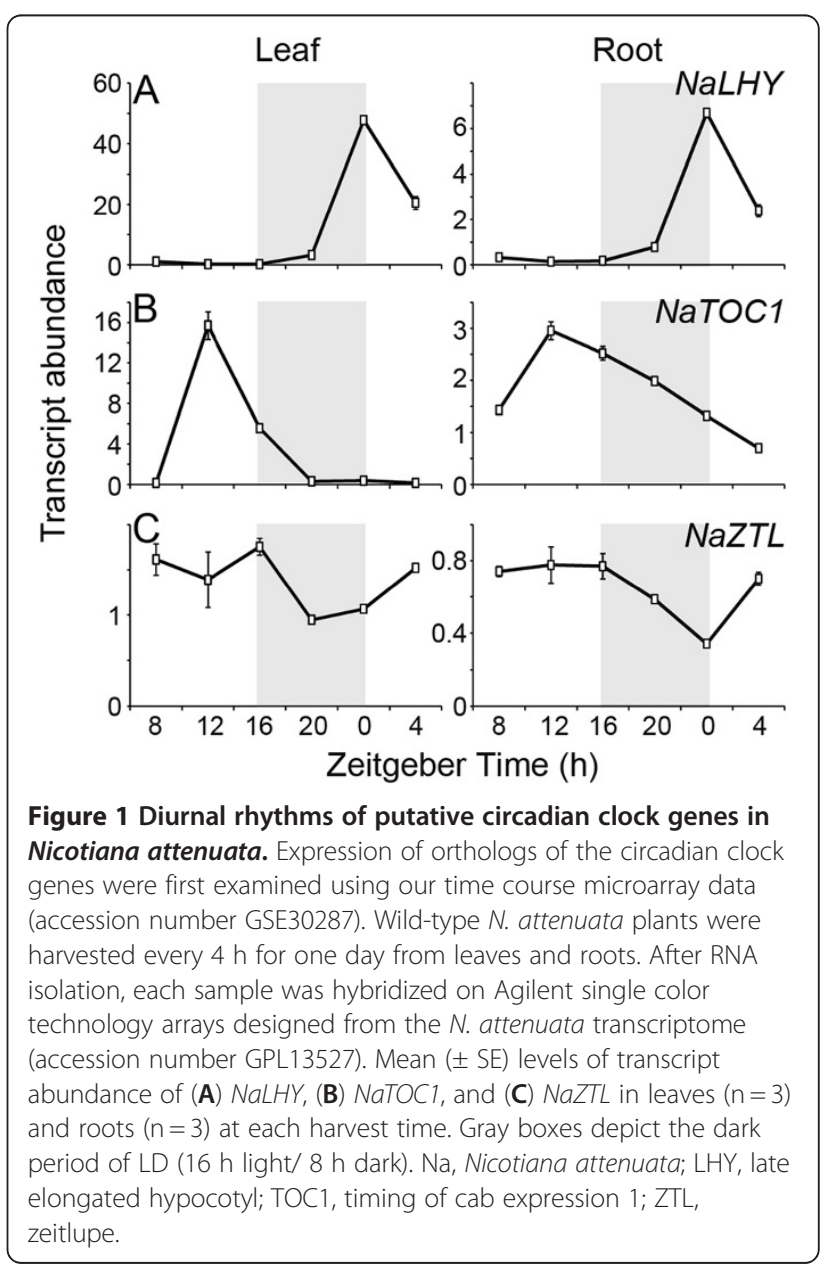


was performed by quantitative real-time PCR (qPCR) using the gene specific primers (Additional file 1). We also constructed full-length of coding sequences of NaLHY, NaTOC1, and NaZTL (Additional file 2), based on the ortholog sequences available in public EST databases and Arabidopsis database. To examine evolutionary relationship of circadian clock genes in plant species, phylogenetic trees were constructed using the UPGMA algorithm (Figure 2 and Additional file 3).

Arabidopsis and poplar genomes contain two MYB transcription factors (LHY/CCA1 and LHY1/2, respectively), which play a key role in the regulation of the endogenous clock [23,36]. However, we found only one oscillating AtLHY-like transcript in our cDNA library (Figure 2A). The transcript levels of NaLHY in LD peaked at Zeitgeber time $0 \mathrm{~h}(\mathrm{ZT} 0 \mathrm{~h})$ and remained low until the next ZT $0 \mathrm{~h}$ (Figures $1 \mathrm{~A}$ and $3 \mathrm{~A}$ ), consistent with the patterns observed in Arabidopsis and rice [26,37]. NaLHY transcript accumulation under LL maintained a circadian rhythm peaking near subjective dawn and showed a shorter period of time with an average $6.1 \mathrm{~h}$ than under LD (Figure 3A). Protein sequence of NaLHY shared a relatively high similarity with AtLHY (Identities $=42 \%$, Positives $=54 \%$, Gaps $=18 \%$ ) and AtCCA1 (Identities $=38 \%$, Positives $=50 \%$, Gaps $=22 \%$ ). Phylogenetic analysis indicated that NaLHY was more closely related to AtLHY than to AtCCA1 (Figure 2). The NaLHY protein (767 aa) is larger than AtLHY (645 aa), AtCCA1 (608 aa), and OsCCA1 (719 aa) but has the conserved SANT domain at the N-terminal and the two alanine rich regions which characterize the AtLHY and OsCCA1 proteins (Additional file 2).

TOC1/PRR1 is a member of the PRR family, which is composed of five oscillating genes; TOC1/PRR1, PRR3, PRR5, PRR7 and PRR9. Each of PRR genes has its own diurnal expression pattern [38]. The microarray data revealed that NaTOC1 transcripts peaked at ZT $12 \mathrm{~h}$ (Figure 1B) in accordance with the known circadian rhythm of the Arabidopsis TOC1 $[39,40]$. The qPCR analysis showed that NaTOC1 transcripts peaked at ZT $12 \mathrm{~h}$ under LD and the expression of NaTOC1 under LL peaked earlier by an average of $1.3 \mathrm{~h}$ compared to the expression under LD (Figure 3B). The full-length NaTOC1 protein sequence exhibited high similarity to AtTOC1/PRR1 (Identities $=49 \%$, Positives $=59 \%$, Gaps $=$ $16 \%)$. Phylogenetic analysis revealed that NaTOC1 is most closely related to AtPRR1 than to other AtPRRs (Figure 2). The REC domain at the $\mathrm{N}$-terminal and the CCT motif in the C-terminal of TOC1 were conserved in $N$. attenuata, Arabidopsis and $O$. sativa but the coiled-coil region was only found in the eudicots Arabidopsis and N. attenuata (Additional file 2).

The Arabidopsis genome encodes three F-box proteins involved in protein degradation of the clock components
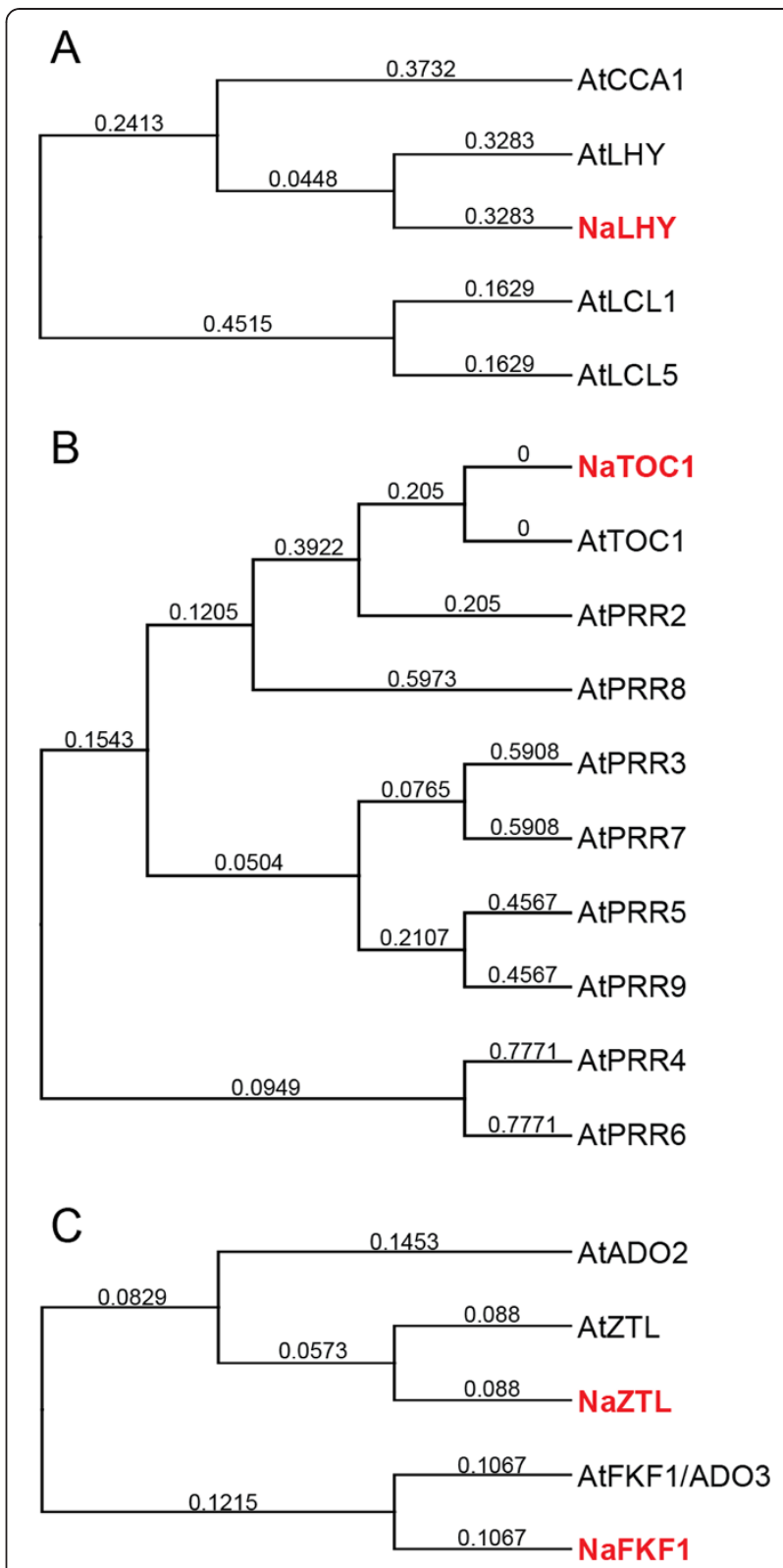

Figure 2 Phylogenetic trees of putative circadian clock genes in N. attenuata. Phylogenetic relationships among predicted orthologs of (A) LHY and CCA1, (B) TOC1 and PRRs, (C) ZTL and FKF1/ADO3 in Arabidopsis thaliana (At) and N. attenuata ( $\mathrm{Na})$. Full-length amino acid sequences were aligned and phylogenetic trees were reconstructed by the UPGMA method. The numbers given for each branch represent the numbers of amino acid substitutions per site. LHY, late elongated hypocotyl; CCA1, circadian clock-associated 1; TOC1, timing of cab expression 1; PRR, pseudo-response regulator; ZTL, zeitlupe; FKF1, flavin-binding kelch repeat F-box 1; ADO, adagio.

$[18,19,21]$. We found two ZTL orthologous genes (NaZTL and NaFKF1/ADO3) in our cDNA library and named them according to the phylogenetic analysis (Figure 2). NaZTL transcripts under LL and LD were largely unchanged (Figures $1 \mathrm{C}$ and $3 \mathrm{C}$ ), consistent with the results from Arabidopsis [41]. In contrast, NaFKF1 


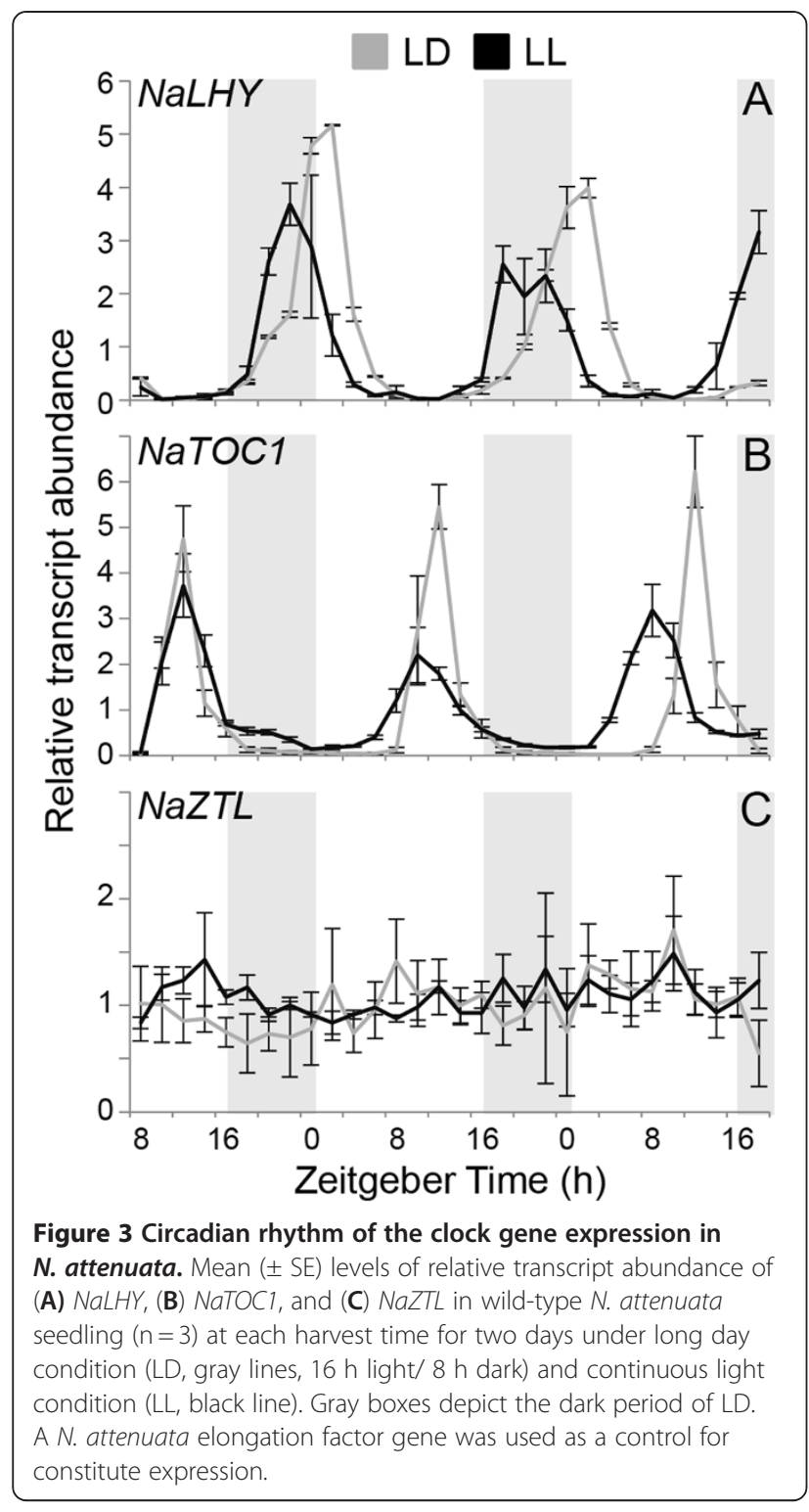

transcripts showed a clear circadian rhythm with peaks at ZT $12 \mathrm{~h}$ under LD (Additional file 4). The period of NaFKF1 expression in LL was shortened by an average of $2 \mathrm{~h}$ (Additional file 4). NaZTL had protein sequence similarity to AtZTL (Identities $=79 \%$, Positives $=86 \%$, Gaps $=5 \%$ ) and NaFKF1 with that of AtFKF1 (Identities = $78 \%$, Positives $=85 \%$, Gaps $=4 \%$ ). NaZTL and NaFKF1 proteins contained LOV/PAS and F-box domains at their N-terminals and Kelch repeats region in their C-terminals as shown in orthologs in Arabidopsis (Additional file 2).

\section{Ectopic expression of $N$. attenuata clock genes in arabidopsis}

To examine the functional conservation of the clock components in N. attenuata, we produced Arabidopsis (Col-0) transgenic plants ectopically expressing $N a L H Y$ and NaZTL genes under the control of Cauliflower Mosaic Virus (CaMV) 35S promoter (35S:NaLHY and 35S: NaZTL). The circadian clock entrains hypocotyl cell elongation to light-dark cycles, and thus plants overexpressing either Arabidopsis LHY or ZTL have elongated hypocotyls compared to wild-type plants [37,42]. We measured the hypocotyl lengths of 35S:NaLHY (Figure 4A) and 35S:NaZTL transgenic plants (Figure 4B). Two independent 35S:NaLHY lines showed a pronounced increase in hypocotyl length compared to wild-type, Col-0, as did the two independent 35S:NaZTL lines $(P<0.05$, one-way ANOVA with Fisher's post hoc test).

The ability to perceive seasonal changes by the circadian clock is required for the successful transition from

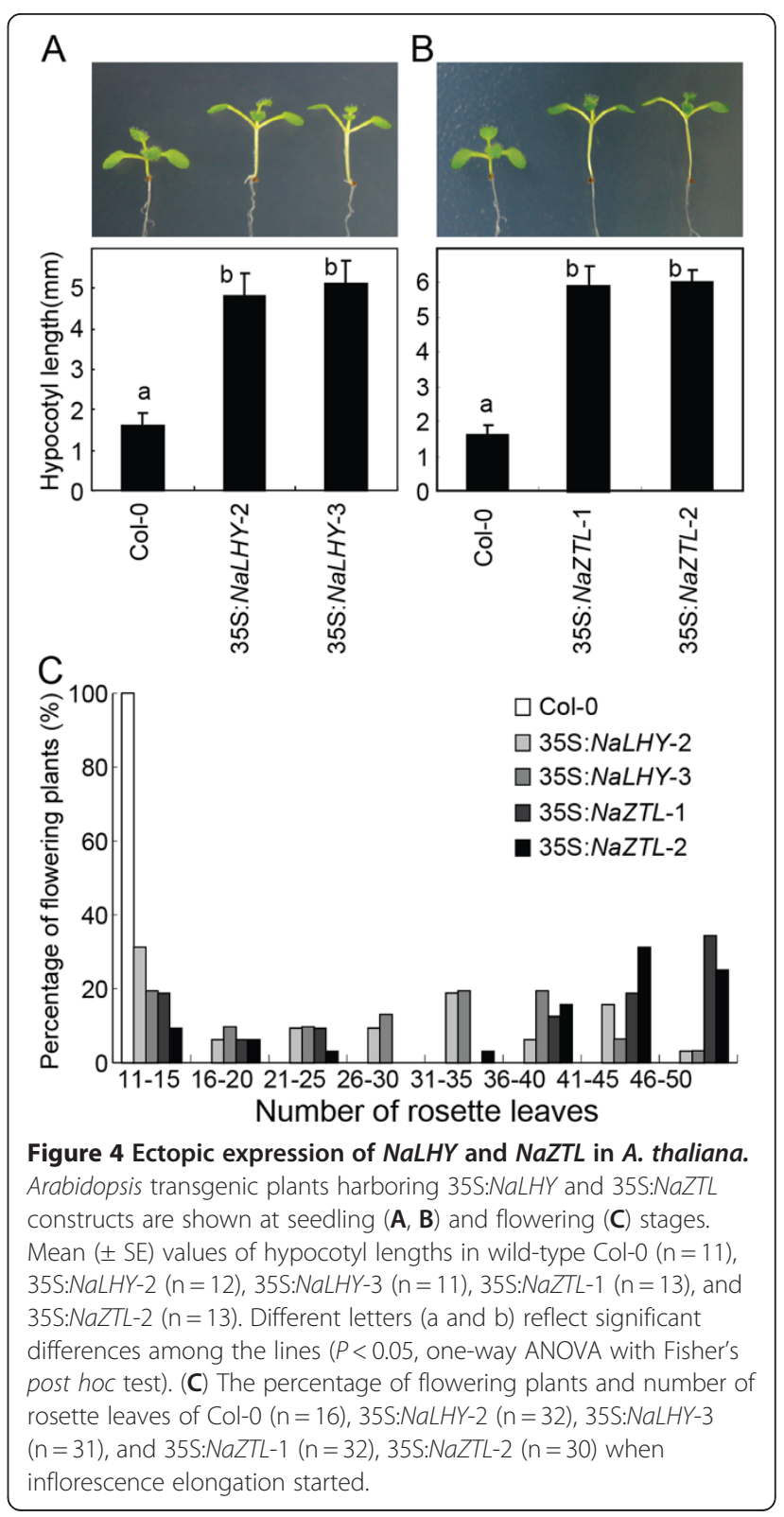


vegetative to reproductive stages. Knocking-out a morning element, Arabidopsis LHY or CCA1 results in an early-flowering phenotype and, in contrast, their overexpressing lines result in a late-flowering phenotype in Arabidopsis [12,36,37]. The overexpression of Arabidopsis ZTL also results in a late-flowering phenotype under LD [42]. To examine the effect of NaLHY and NaZTL on flowering time regulation in Arabidopsis, we scored the rosette leaf number at a time when initial flowering was observed in plants grown under LD. Both 35S:NaLHY and 35S:NaZTL plants exhibited delayed flowering phenotypes and increased rosette leaf numbers (Figure $4 \mathrm{C}$ ).

\section{Interaction between NaTOC1 and NaZTL protein}

F-box protein ZTL plays a key role in protein turnover of the clock components, including TOC1 by direct proteinprotein interactions [18]. We determined whether NaTOC1 interacts with NaZTL by yeast two-hybrid analysis. In addition, we tested inter-species interactions of AtTOC1NaZTL and AtZTL-NaTOC1. Full-length NaTOC1 proteins (AtTOC1) fused with the GAL4 activation domain (AD), and NaZTL (AtZTL) fused with the GAL4 binding domain (BD) were co-expressed in yeast cells (see Methods). The yeast cells expressing both NaTOC1 and
NaZTL constructs grew well in the absence of leucine, tryptophan, histidine, and adenine, showing the positive interaction, consistent with Arabidopsis TOC1 and ZTL interaction (Figure 5A). The transformants expressing both NaTOC1 and NaZTL also exhibited strong $\beta$-galactosidase activity compared to control yeast cells carrying empty vectors or only one of the constructs (Figure 5B). Interspecies protein interactions of AtTOC1-NaZTL and AtZTL-NaTOC1 were as strong as the intra-species interactions of TOC1 and ZTL (Figure 5).

\section{Silencing of NaTOC1 in N. attenuata}

To examine the conserved and unique functions of circadian clock genes in $N$. attenuata, we first silenced NaTOC1 expression by constitutive overexpression of NaTOC1-specific inverted repeat (ir) sequences (irTOC1 lines) $[43,44]$. TOC1 in Arabidopsis plays a key role in flowering time regulation. The semi-dominant toc1-1 mutant displays a late-flowering phenotype under LD and an early-flowering phenotype under short day conditions (SD) [39] and the toc1-2 loss-of-function mutant shows no significant difference of flowering time under LD but an early-flowering phenotype under SD [45]. During the screening of the silenced lines, we clearly observed
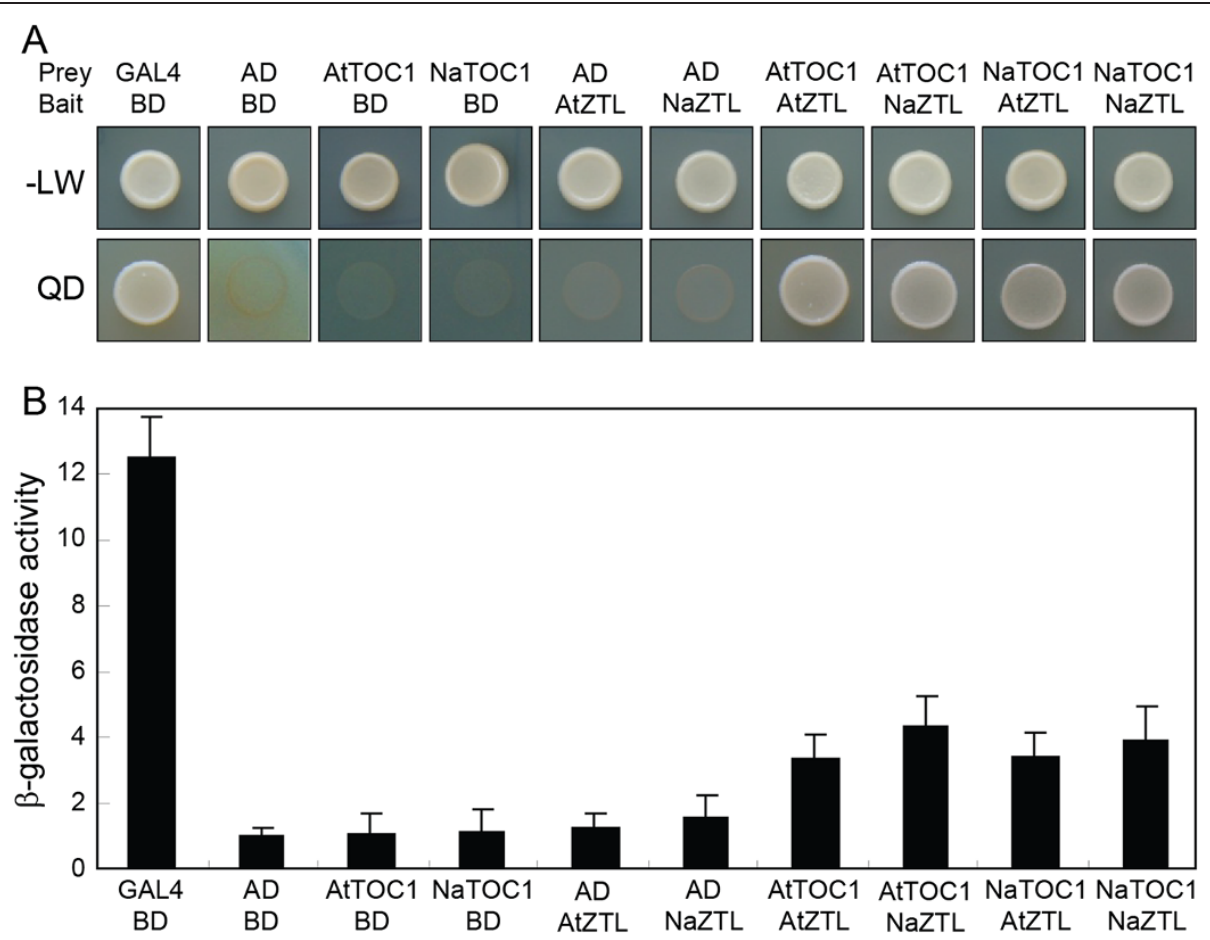

Figure 5 Protein interactions between TOC1 and ZTL from N. attenuata and A. thaliana using a yeast two-hybrid assay. (A) Growth of the yeast cells carrying prey and bait constructs indicated on the top of each panel. The ZTL proteins were fused to the GAL4 DNA binding domain (bait), and the TOC1 proteins were fused to the GAL4 activation domain (prey). The yeast cells can grow on QD medium when bait and prey proteins physically bind. (B) Mean ( \pm SE) levels of $\beta$-galactosidase activity of the yeast carrying prey and bait constructs. BD, empty vector expressing binding domain; AD, empty vector expressing activation domain; -LW, synthetic dropout (SD) yeast growth medium lacking leucine and tryptophan; QD, SD medium lacking Leu, Trp, histidine, and adenine. 
the late-flowering phenotypes of several independent irTOC1 lines under LD (Figure 6). Two independent lines were used to measure flowering time and rosette leaf number when the first flower opened (Figure 6C). The silencing efficiency of independent lines harboring the RNAi construct correlated strongly with the flowering phenotype (Figure 6B). In one homozygous irTOC1162 line, screened on the basis of antibiotic resistance, but in which the TOC1 gene expression was not silenced, presumably due to methylation of the ir part of the T-DNA or an unknown insertion effect, displayed a WT flowering pattern (Figure 6).

\section{Discussion}

Since the clock genes of Arabidopsis have been identified, the clock mechanism of Arabidopsis has been extended to other dicotyledonous plants; soybean [24], chestnut [29], Brassica rapa [46], poplar [23] and also to monocotyledonous plants; rice [25], maize [22], duckweed [28]. Here, we identified three core circadian clock genes in a wild tobacco, $N$. attenuata. Analyses of the circadian rhythms of transcript accumulation and protein similarity allowed us to identify orthologs of $L H Y, T O C 1$, and ZTL of Arabidopsis in N. attenuata. Protein interactions of TOC1 and ZTL in Arabidopsis were also conserved in $N$. attenuata. In addition, ectopic expression of NaLHY and NaZTL in Arabidopsis confirmed the functional conservation of LHY and ZTL in N. attenuata. We also demonstrated that NaTOC1 in N. attenuata plays an important role in the regulation of flowering time.

The number of $L H Y$ (or CCA1) orthologous genes and the timing of gene duplication events differ among plant species [23]. One common ancestor of LHY/CCA1 independently duplicated in monocots and eudicots. Populus nigra and $P$. trichocarpa contain two $L H Y$ orthologs and the monocots, rice and Sorghum bicolor contain one CCA1-like gene in their genome [23,47]. Gene duplication events of LHYICCA1 in popular and Arabidopsis would be expected in $N$. attenuata but we were able to find only one $L H Y / C C A 1$ like gene in $N$. attenuata. However, the
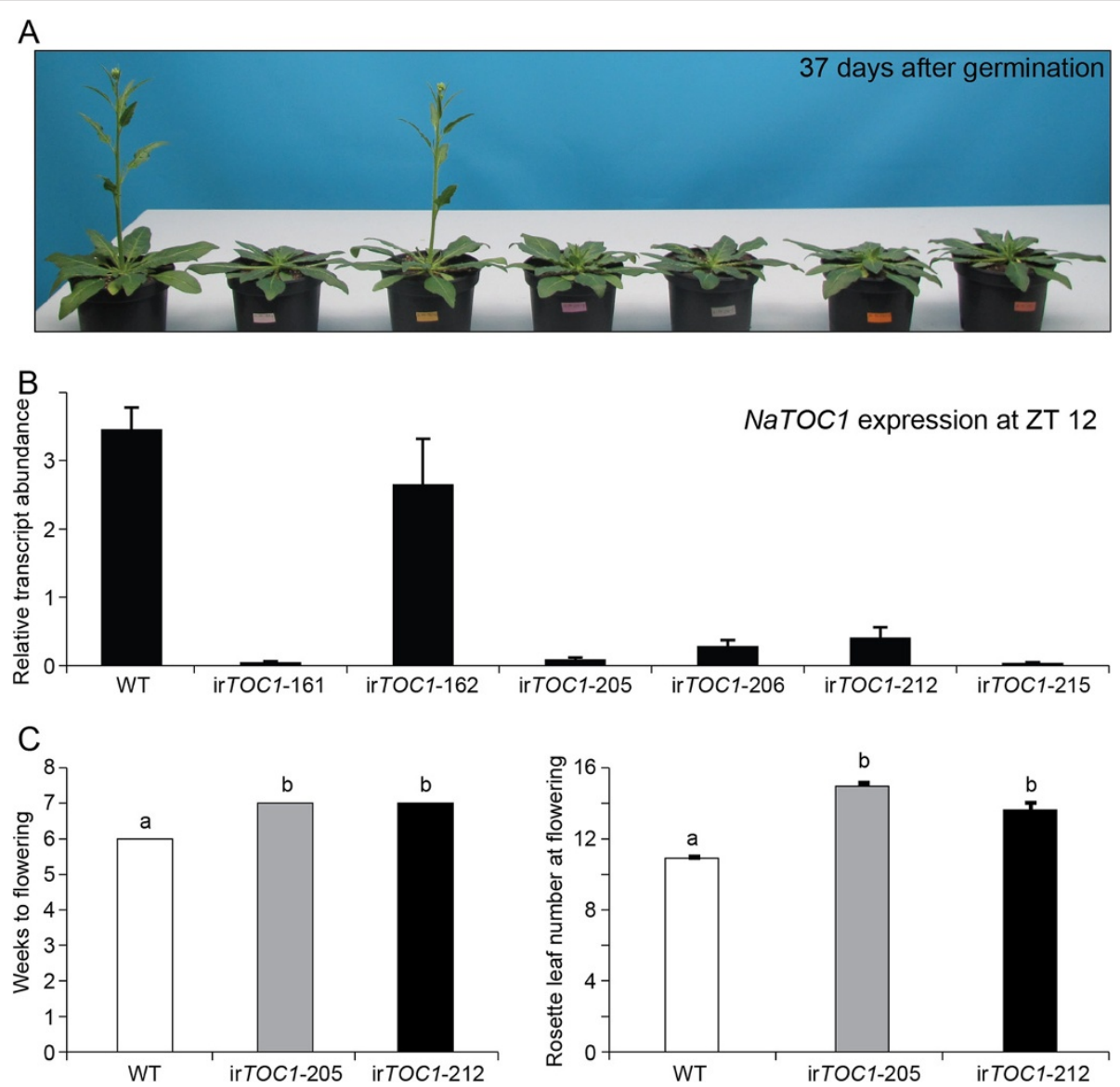

Figure 6 Late-flowering phenotypes of NaTOC1 silenced N. attenuata. (A) Phenotypes of transgenic plants silenced in NaTOC1 gene expression (irTOC1) with an inverted repeat (ir) RNAi construct. (B) Mean ( \pm SE) levels of NaTOC1 $(n=3)$ transcript accumulation at zeitgeber time (ZT) 12 in wild-type and in several irTOC1 lines. (C) Time on weeks of WT and 2 selected irTOC1 lines to reach flowering stage $(n=5)$. (D) Mean $( \pm$ SE) number of leaves at flowering stage $(n=5)$. 
second ortholog could be missing in our current cDNA library and we plan to perform deep sequencing of the transcriptome and microarray analysis of plants grown under a variety of conditions in the future to clarify the evolutionary relationship of $N$. attenuata's $L H Y$ orthologs.

While components of the endogenous clock and their associated circadian clock mechanisms have clearly been maintained across diverse plant species, clock-mediated signaling has evolved in response to differing selection pressures. A perennial plant, chestnut (Castanea sativa) has orthologs of AtLHY and AtTOC1 in its genome and the pattern of transcript accumulation of CsLHY and CsTOC1 is similar to that of Arabidopsis in LD at $22^{\circ} \mathrm{C}$ [29]. However, in winter condition, both CsLHY and CsTOC1 transcripts lose their diurnal rhythms and maintain high levels of transcripts, which may be associated with the induction of winter dormancy [29]. Even within a single species, A. thaliana, genetic variation in the clock components plays a critical role in adapting ecotypes to their local environment [48]. Genetic variation in the $P R R$ genes is associated with local adaptation seen in the differential expression of quantitative trait loci in 150 Arabidopsis ecotypes [48]. In addition, the degree to which TOC1 regulates flowering time differs among Arabidopsis ecotypes. A semi-dominant toc1-1 mutant of the Arabidopsis C24 ecotype displays late a flowering phenotype in LD, whereas the same TOC1 mutation in the Landsberg ecotype results in no change in flowering time compared with WT plants under LD [39]. The toc1-2 mutant shows also no change in flowering time under LD [45]. However, silencing TOC1 in $N$. attenuata confers a late-flowering phenotype under LD, which may be due to the longer life span of $N$. attenuata (about 3 months) or different circadian clock functions have evolved under various environmental pressures. In future research, we plan to measure the flowering time of irTOC1 lines under SD to examine the light sensitivity of this transgenic line.

We have investigated the ecology of $N$. attenuata in its native habitat for more than twenty years. During this period, we have observed interesting diurnal rhythmic traits of N. attenuata and time-of-day dependent ecological interactions. For example, $N$. attenuata interacts with different groups of herbivores which are either dayactive (such as grasshoppers, mirids and Manduca larvae) or night-active (such as noctuid larvae and tree crickets) and produces different chemicals that function as direct defenses against these herbivores or function as indirect defenses and attract of predators of the herbivores [49]. Recently, we showed that tissue specific diurnal rhythm of metabolites and its related transcripts in $N$. attenuata changes in response to herbivore attack of a specialist, $M$. sexta larvae [35]. More than $15 \%$ of total metabolites that we measured in leaf and root shows diurnal patterns and some of them have been demonstrated to function as plant defenses against herbivore attack. Goodspeed et al. [50] have recently reported that feeding behavior of Tricoplusia $n i$ is predicted by the circadian clock in its host plant Arabidopsis and it increases anti-herbivore defense of Arabidopsis. All of these interactions provide a rich arena in which to explore the molecular mechanism of how the circadian clock regulates plant-insect interactions.

\section{Conclusions}

We identified three core circadian clock components in $N$. attenuata based on the gene expression and phenotypic alterations in lines silenced or overexpressed in the components. This work provides the foundation for the manipulation the ecological roles of the circadian clock in nature. As jet travel has revealed the depth of circadianregulated processes in humans, circadian mutants of $N$. attenuata will be used to unravel the ecological functions of the clock in plant-environment, plant-plant, and plant-insect interactions in nature.

\section{Methods}

\section{Plant growth condition}

For all experiments we used Nicotiana attenuata Torr. Ex. Wats (Solanaceae) plants ( $31^{\text {st }}$ inbred generation), wild-type (WT) originating from a population in Utah. Seeds were sterilized and germinated on Petri Dishes with Gamborg's B5 media as described in [43]. Petri dishes with 20 seeds were kept under long day conditions (LD, $16 \mathrm{~h}$ light/ $8 \mathrm{~h}$ dark).

To examine the free running period, one group of seedlings 15 days after germination was moved into constant light conditions for the 3 days of sampling and the other group of seedlings was grown under LD. Three biological replicates (10 seedlings pooled per replicate) were harvested every $2 \mathrm{~h}$ for 3 days and immediately frozen in liquid nitrogen.

\section{RNA isolation and gene expression}

Total RNA was extracted using TRIZOL reagent and cDNA was synthesized from 500 ng of total RNA using RT-minus kit (Fermentas, Burlington, Canada). The quantitative RT-PCR analyses were performed on a Stratagene MX3005P (Agilent Technologies, Santa Clara, CA, USA) employing SYBR Green kits (Eurogentec, Cologne, Germany). Primers were designed based on sequences from Arabidopsis thaliana retrieved from TAIR website and a $N$. attenuata transcript library (NCBI GEO Database accession number GSE30287). The sequences of qRT-PCR primer pairs for NaLHY, NaTOC1, NaZTL and NaFKF1 are listed in Additional file 1. Transcript abundance expressed relative to the expression of $N$. attenuata ELONGATION FACTOR gene. 


\section{Phylogenetic analysis}

The identity of the circadian genes NaLHY (NCBI accession number JQ424913), NaTOC1 (Accession number JQ424914), NaZTL (Accession number JQ424912) and NaFKF1/ADO3 (Accession number JQ424915) was determined by sequencing. A standard PCR was performed to obtain the full length cDNA and the list of primers used for this analysis is in Additional file 1. PCR products were subcloned for amplification using a CloneJET PCR Cloning kit (Fermentas).

The amino acid sequences of the circadian genes NaLHY, NaTOC1, NaZTL and NaFKF1/ADO3 were deduced from cDNA sequences and aligned using the Geneious program V5.3 (http://www.geneious.com). The numbers of amino acid substitutions were estimated by a Jukes-Cantor model using a BLOSUM 62 matrix, through a global alignment with free end gaps option. A phylogenetic tree was reconstructed by the Unweighted Pair Group Method with Arithmetic Mean (UPGMA) method. These analyses were performed using Geneious software V5.3.

\section{Overexpression of NaLHY and NaZTL in arabidopsis}

All $A$. thaliana lines used were in the Col-0 background. Plants were grown in a controlled culture room at $22^{\circ} \mathrm{C}$ with a relative humidity of 55\% under long-day (LD) conditions (16-h light/8-h dark) with white light illumination $\left(120 \mu \mathrm{mol}\right.$ photons $\left./ \mathrm{m}^{2} \mathrm{~s}\right)$ provided by fluorescent FLR40D/A tubes (Osram). To produce transgenic plants overexpressing the $N a L H Y$ and $N a Z T L$ genes, full-length cDNAs were subcloned into the binary pB2GW7 vector under the control of the CaMV $35 \mathrm{~S}$ promoter (Invitrogen, Carlsbad, CA, USA). Agrobacterium tumefaciensmediated Arabidopsis transformation was performed according to a modified floral dip method (Clough and Bent, 1998). $T_{2}$ transgenic plants harboring a single T-DNA insertion were used in subsequent assays including hypocotyl length and flowering time measurements. Transformation with NaTOC1 was carried out but for unknown technical reasons we were not possible to regenerate plants from it. Selected lines were checked by RT-PCR for the NaLHY and NaZTL overexpression (Additional file 5), using A. thaliana tubulin (TUB) as a reference gene.

\section{Yeast-two-hybrid}

Yeast two-hybrid assays were performed using the Matchmaker ${ }^{\mathrm{TM}}$ system (Clontech, Palo Alto, CA, USA). Primers used for amplifying NaTOC1, AtTOC1, NaZTL, and AtZTL were described in Additional file 1. Each RT-PCR product was digested with restriction enzymes (EcoRI, XmaI for NaTOC1 and AtTOC1, NcoI, XmaI for NaZTL, NdeI, BamHI for AtZTL). Digested full-length transcripts of TOC1s and ZTLs were subcloned into
pGADT7 and pGBKT7, respectively. The yeast strain AH109 (leucine-, tryptophan-, histidine-, adenine-) contained lac Z reporter gene was co-transformed with the indicated vectors in Figure 5A. Transformation was conducted according to the manufacturer's instructions (Clontech). Single colonies obtained on growth medium lacking Leu, Trp were inoculated on a medium without Leu, Trp, His, Ade and used to measure $\beta$-galactosidase activity described in the instructions (Clontech).

\section{Silencing of NaTOC1 in N. attenuata}

A sequence fragment of $\mathrm{NaTOC1}$ cDNA was inserted into the pSOL8 transformation vector as an inverted repeat construct driven by the CaMV $35 \mathrm{~S}$ promoter $[43,44]$. The NaTOC1 vector was transformed into N. attenuata WT plants using Agrobacterium tumefaciens mediated transformation; ploidy was determined on $\mathrm{T}_{0}$ plants as described by Gase et al. [44], allowing for the selection of only diploid transformed lines. Homozygosity was confirmed on $\mathrm{T}_{2}$ plants by hygromycin resistance screening after which 6 transformed lines were selected and transferred to the glasshouse for further growth at conditions described in Krügel et al. [43]. Gene expression levels of NaTOC1 were determined by qPCR from tissue of selected $\mathrm{T}_{2}$ plants and wild-type plants collected at ZT $12 \mathrm{~h}$.

\section{Additional files}

Additional file 1: List of primers used for transcript profiling and full-length cloning of circadian clock genes in N. attenuata and $A$. thaliana.

Additional file 2: Protein alignments of circadian clock genes in $\mathbf{N}$. attenuata, Arabidopsis and rice. Full-length amino acid sequences were aligned using the Geneious software.

Additional file 3: Phylogenetic trees of circadian clock genes in several plant species. Phylogenetic trees of (A) LHY/CCA1, (B) TOC1, (C) ZTL and (D) FKF1/ADO3. Full-length amino acid sequences were aligned using the Geneious software. Phylogenetic trees were constructed with available sequences from 3 major taxonomical groups: eudicots, monocots and one moss Selaginella moellendorffii for TOC1 and ZTL trees. Unweighted Pair Group Method with the Arithmetic Mean (UPGMA) method from the numbers of amino acid substitutions by applying the Jukes-Cantor model was used. The scale bar represents the number of amino acid substitutions per site. Ac, Allium cepa; At,

Arabidopsis thaliana; Cs, Castanea sativa; Gm, Glycine max; In, Ipomoea nil; Mc, Mesembryanthemum crystallinum; Na, Nicotiana attenuata; Os, Oryza sativa; Pn, Populus nigra; Pt, Populus trichocarpa; Pv, Phaseolus vulgaris; Sb, Sorghum bicolor; SI, Solanum lycopersicum; Sm, Selaginella moellendorffii; $\mathrm{V}$, Vitis vinifera; Ta, Triticum aestivum; Zm, Zea mays.

Additional file 4: Circadian rhythm of the NaFKF1/ADO3 expression in N. attenuata. (A) Mean ( \pm SE) levels of transcript abundance of NaFKF1/ADO3 were examined using our time course microarray data (accession number GSE30287). Wild-type N. attenuata plants were harvested every $4 \mathrm{~h}$ for one day from leaves $(n=3)$ and roots $(n=3)$. After RNA isolation, each sample was hybridized on Agilent single color technology arrays designed from the $N$. attenuata transcriptome (accession number GPL13527). (B) Mean ( \pm SE) levels of relative transcript abundance of NaFKF1/ADO3 in wild-type $N$. attenuata seedling $(n=20)$ at each harvest time for two days under long day condition (LD, gray lines, 
$16 \mathrm{~h}$ light/ $8 \mathrm{~h}$ dark) and continuous light condition (LL, black line). Transcript levels were determined by GPCR and N. attenuata Elongation Factor was used as reference gene. Gray boxes depict the dark period of LD.

\section{Additional file 5: Overexpression of $\mathrm{NaLHY}$ and NaZTL transcripts in} Arabidopsis transformants. Transcript levels were measured by RT-PCR. Primers (see Additional file 1) were designed for the specific detection of NaLHY or NaZTL transcripts, not for Arabidopsis LHY or ZTL. Plants grown under LD were harvested at ZT $8 \mathrm{~h}$. A tubulin gene (TUB) was included as a control.

\section{Authors' contributions}

FY, PS, SK designed experiments and carried out the lab work, JR transformed 35S:NaLHY and 35S:NaZTL construct into Arabidopsis, CP, ITB and SK conceived the project and oversaw the research. All authors wrote, read and approved the final manuscript.

\section{Acknowledgements}

We thank the Max Planck Society for financial support. This work was also supported by the Leaping Research Program (20110016440) provided by the National Research Foundation of Korea, the Next-Generation BioGreen 21 program (Plant Molecular Breeding Center No. PJ008103) provided by the Rural Development Administration, and by grants from the Plant Signaling Network Research Center (20110001099), the National Research Foundation of Korea (20110027355), and the Agricultural R \& D Promotion Center (309017-03), Korea Ministry for Food, Agriculture, Forestry and Fisheries.

\section{Author details}

'Department of Molecular Ecology, Max Planck Institute for Chemical Ecology, Hans-Knöll-Straße 8, Jena D-07745, Germany. ${ }^{2}$ Department of Chemistry, Seoul National University, Seoul 151-742, Korea. ${ }^{3}$ Department of Chemistry, Chonbuk National University, Jeonju 561-756, Korea.

Received: 23 January 2012 Accepted: 22 September 2012

Published: 25 September 2012

\section{References}

1. Dunlap JC: Genetic and molecular analysis of circadian rhythms. Annu Rev Genet 1996, 30(1):579-601.

2. Sanchez A, Shin J, Davis SJ: Abiotic stress and the plant circadian clock. Plant Signaling Behav. 2011, 6(2):223-231.

3. Yakir E, Hilman D, Harir Y, Green RM: Regulation of output from the plant circadian clock. FEBS J 2007, 274(2):335-345.

4. Barak S, Tobin EM, Green RM, Andronis C, Sugano S: All in good time: the Arabidopsis circadian clock. Trends Plant Sci 2000, 5(12):517-522.

5. Golden SS, Canales SR: Cyanobacterial circadian clocks - timing is everything. Nat Rev Microbiol 2003, 1(3):191-199.

6. Lombardi LM, Brody S: Circadian rhythms in neurospora crassa: clock gene homologues in fungi. Fungal Genet Biol 2005, 42(11):887-892.

7. Panda S, Antoch MP, Miller BH, Su Al, Schook AB, Straume M, Schultz PG, Kay SA, Takahashi JS, Hogenesch JB: Coordinated transcription of key pathways in the mouse by the circadian clock. Cell 2002, 109(3):307-320.

8. Albrecht U, Eichele G: The mammalian circadian clock. Curr Opin Genet Dev 2003, 13(3):271-277.

9. Loros JJ, Dunlap JC: Genetic and molecular analysis of circadian rhythms in N. eurospora. Annu Rev Physiol 2001, 63(1):757-794.

10. Williams JA, Sehgal A: Molecular components of the circadian system in drosophila. Annu Rev Physiol 2001, 63(1):729-755.

11. Ueda HR: Systems biology flowering in the plant clock field. Mol Syst Biol 2006, 2:60.

12. Wang Z-Y, Tobin EM: Constitutive expression of the CIRCADIAN CLOCK ASSOCIATED 1 (CCA1) gene disrupts circadian rhythms and suppresses its own expression. Cell 1998, 93(7):1207-1217.

13. Gendron JM, Pruneda-Paz JL, Doherty CJ, Gross AM, Kang SE, Kay SA Arabidopsis circadian clock protein, TOC1, is a DNA-binding transcription factor. Proc Natl Acad Sci USA 2012, 109(8):3167-3172.

14. Pokhilko A, Fernández AP, Edwards KD, Southern MM, Halliday K, Millar AJ: The clock gene circuit in Arabidopsis includes a repressilator with additional feedback loops. Mol Syst Biol 2012, 8:574.
15. Alabadí D, Oyama T, Yanovsky MJ, Harmon FG, Más P, Kay SA: Reciprocal regulation between TOC1 and LHY/CCA1 within the Arabidopsis circadian clock. Science 2001, 293(5531):880-883.

16. Farré EM, Harmer SL, Harmon FG, Yanovsky MJ, Kay SA: Overlapping and distinct roles of PRR7 and PRR9 in the Arabidopsis circadian clock. Curr Biol 2005, 15(1):47-54

17. Salomé PA, McClung CR: PSEUDO-RESPONSE REGULATOR 7 and 9 are partially redundant genes essential for the temperature responsiveness of the Arabidopsis circadian clock. Plant Cell 2005, 17(3):791-803.

18. Mas P, Kim W-Y, Somers DE, Kay SA: Targeted degradation of TOC1 by ZTL modulates circadian function in Arabidopsis thaliana. Nature 2003, 426(6966):567-570.

19. Imaizumi T, Schultz TF, Harmon FG, Ho LA, Kay SA: FKF1 F-box protein mediates cyclic degradation of a repressor of CONSTANS in Arabidopsis. Science 2005, 309(5732):293-297.

20. Sawa M, Nusinow DA, Kay SA, Imaizumi T: FKF1 and GIGANTEA complex formation is required for day-length measurement in Arabidopsis. Science 2007, 318(5848):261-265.

21. Baudry A, Ito S, Song YH, Strait AA, Kiba T, Lu S, Henriques R, Pruneda-Paz $J \mathrm{~L}$, Chua N-H, Tobin EM, et al: F-box proteins FKF1 and LKP2 act in concert with ZEITLUPE to control Arabidopsis clock progression. Plant Cell 2010, 22(3):606-622.

22. Wang X, Wu L, Zhang S, Ku L, Wei X, Xie L, Chen Y: Robust expression and association of ZmCCA1 with circadian rhythms in maize. Plant Cell Rep 2011, 30(7):1261-1272.

23. Takata N, Saito S, Tanaka Saito C, Nanjo T, Shinohara K, Uemura M: Molecular phylogeny and expression of poplar circadian clock genes, LHY1 and LHY2. New Phytol 2009, 181(4):808-819.

24. Liu H, Wang H, Gao P, Xu J, Xu T, Wang J, Wang B, Lin C, Fu YF: Analysis of clock gene homologs using unifoliolates as target organs in soybean (Glycine max). J Plant Physiol 2009, 166(3):278-289.

25. Murakami M, Tago Y, Yamashino T, Mizuno T: Comparative overviews of clock-associated genes of arabidopsis thaliana and oryza sativa. Plant Cell Physiol 2007, 48(1):110-121.

26. Izawa T, Oikawa T, Sugiyama N, Tanisaka T, Yano M, Shimamoto K Phytochrome mediates the external light signal to repress FT orthologs in photoperiodic flowering of rice. Genes Dev 2002, 16(15):2006-2020.

27. Kaldis A-D, Kousidis P, Kesanopoulos K, Prombona A: Light and circadian regulation in the expression of $L H Y$ and Ihcb genes in phaseolus vulgaris. Plant Mol Biol 2003, 52(5):981-997.

28. Miwa K, Serikawa M, Suzuki S, Kondo T, Oyama T: Conserved expression profiles of circadian clock-related genes in two lemna species showing long-day and short-day photoperiodic flowering responses. Plant Cell Physiol 2006, 47(5):601-612

29. Ramos A, Pérez-Solís E, lbánezez C, Casado R, Collada C, Gómez L, Aragoncillo C, Allona I: Winter disruption of the circadian clock in chestnut. Proc Nat Acad Sci USA 2005, 102(19):7037-7042.

30. Taylor A, Massiah AJ, Thomas B: Conservation of arabidopsis thaliana photoperiodic flowering time genes in onion (allium cepa L.). Plant Cell Physiol 2010, 51(10):1638-1647.

31. Xue ZG, Zhang XM, Lei CF, Chen XJ, Fu YF: Molecular cloning and functional analysis of one ZEITLUPE homolog GmZTL3 in soybean. Mol Biol Rep 2011, 39(2):1411-1418.

32. Dodd AN, Salathia N, Hall A, Kévei E, Tóth R, Nagy F, Hibberd JM, Millar AJ, Webb AAR: Plant circadian clocks increase photosynthesis, growth, survival, and competitive advantage. Science 2005, 309(5734):630-633.

33. Yerushalmi S, Yakir E, Green RM: Circadian clocks and adaptation in Arabidopsis. Mol Ecol 2011, 20(6):1155-1165.

34. Green RM, Tingay S, Wang ZY, Tobin EM: Circadian rhythms confer a higher level of fitness to Arabidopsis plants. Plant Physio/ 2002, 129(2):576-584.

35. Kim SG, Yon F, Gaquerel E, Gulati J, Baldwin IT: Tissue specific diurnal rhythms of metabolites and their regulation during herbivore attack in a native tobacco. Nicotiana attenuata. PLoS One 2011, 6(10):e26214.

36. Mizoguchi T, Wheatley K, Hanzawa Y, Wright L, Mizoguchi M, Song H-R, Carré IA, Coupland G: LHY and CCA1 are partially redundant genes required to maintain circadian rhythms in Arabidopsis. Dev Cell 2002, 2(5):629-641.

37. Schaffer R, Ramsay N, Samach A, Corden S, Putterill J, Carré IA, Coupland G: The late elongated hypocotyl mutation of arabidopsis disrupts circadian rhythms and the photoperiodic control of flowering. Cell 1998, 93(7):1219-1229. 
38. Matsushika A, Makino S, Kojima M, Mizuno T: Circadian waves of expression of the APRR1/TOC1 family of pseudo-response regulators in arabidopsis thaliana: insight into the plant circadian clock. Plant Cell Physiol 2000, 41(9):1002-1012.

39. Somers DE, Webb A, Pearson M, Kay SA: The short-period mutant, toc1-1, alters circadian clock regulation of multiple outputs throughout development in arabidopsis thaliana. Development 1998, 125(3):485-494.

40. Millar AJ, Carre IA, Strayer CA, Chua NH, Kay SA: Circadian clock mutants in arabidopsis identified by luciferase imaging. Science 1995, 267(5201):1161.

41. Somers DE, Schultz TF, Milnamow M, Kay SA: ZEITLUPE encodes a novel clock-associated PAS protein from arabidopsis. Cell 2000, 101(3):319-329.

42. Somers DE, Kim WY, Geng R: The F-box protein ZEITLUPE confers dosage-dependent control on the circadian clock, photomorphogenesis, and flowering time. Plant Cell 2004, 16(3):769-782.

43. Krügel T, Lim M, Gase K, Halitschke R, Baldwin IT: Agrobacterium -mediated transformation of Nicotiana attenuata, a model ecological expression system. Chemoecology 2002, 12(4):177-183.

44. Gase K, Weinhold A, Bozorov T, Schuck S, Baldwin IT: Efficient screening of transgenic plant lines for ecological research. Mol Ecol Resour 2011, 11(5):890-902.

45. Niwa Y, Ito S, Nakamichi N, Mizoguchi T, Niinuma K, Yamashino T, Mizuno T: Genetic linkages of the circadian clock-associated genes, TOC1, CCA1 and $L H Y$, in the photoperiodic control of flowering time in Arabidopsis thaliana. Plant Cell Physiol 2007, 48(7):925-937.

46. Xu X, Xie Q, McClung CR: Robust circadian rhythms of gene expression in brassica rapa tissue culture. Plant Physiol 2010, 153(2):841-850.

47. McClung CR: A modern circadian clock in the common angiosperm ancestor of monocots and eudicots. BMC Biol 2010, 8(1):55.

48. Michael TP, Salomé PA, Yu HJ, Spencer TR, Sharp EL, McPeek MA, Alonso $J M$, Ecker JR, McClung CR: Enhanced fitness conferred by naturally occurring variation in the circadian clock. Science 2003, 302(5647):1049.

49. Wu J, Baldwin IT: New insights into plant responses to the attack from insect herbivores. Annu Rev Genet 2010, 44:1-24.

50. Goodspeed D, Chehab EW, Min-Venditti A, Braam J, Covington MF: Arabidopsis synchronizes jasmonate-mediated defense with insect circadian behavior. Proc Natl Acad Sci U S A 2012, 109(12):4674-4677.

doi:10.1186/1471-2229-12-172

Cite this article as: Yon et al: Identification and characterization of circadian clock genes in a native tobacco, Nicotiana attenuata. BMC Plant Biology 2012 12:172.

\section{Submit your next manuscript to BioMed Central and take full advantage of:}

- Convenient online submission

- Thorough peer review

- No space constraints or color figure charges

- Immediate publication on acceptance

- Inclusion in PubMed, CAS, Scopus and Google Scholar

- Research which is freely available for redistribution 